the report was for a Quality Improvement Project to be carried out in order to formalise handover.

The handover procedure at Chase Farm Hospital for core trainee doctors 'on-call' prior to this QIP was not standardised and consisted of an informal, verbal handover. Frustrations had been raised by doctors and other staff members that this current method of handover was unreliable and unsafe.

Method. We sent out a questionnaire about handover to all doctors on the on-call rota to help establish what intervention would be appropriate.

We then performed a retrospective collection of documented handovers within a two month time period.

Our intervention was to introduce an email handover procedure.

Following a two month trial of this intervention, we resent the questionnaire and performed a second retrospective collection of handover documentation.

Result. Prior to this QIP we found that $0 \%$ of on call handovers were being formally documented. After the introduction of our handover email $88 \%$ of handovers were being formally documented using the handover email.

Satisfaction with the handover procedure went from $0 \%$ being very satisfied and only $33 \%$ being satisfied to $50 \%$ being satisfied and $50 \%$ being very satisfied.

Conclusion. A standardised and documented handover procedure is crucial for patient safety and to allow doctors to communicate jobs effectively with each other.

A secure email for handover is a successful way of formalising the handover process.

Limitations include:

Access to the handover email for new staff or locum staff.

Ensuring that doctors who aren't on the on-call rota know how to use it to handover their ward jobs.

\section{Rehabilitation during a pandemic: psychiatrists as first responders?}

Jeremy Cave ${ }^{1 \star}$ and Matthieu Crews ${ }^{2}$

${ }^{1}$ Core Trainee, South London and Maudsley NHS Foundation Trust and ${ }^{2}$ South London and Maudsley NHS Foundation Trust

${ }^{*}$ Corresponding author.

doi: 10.1192/bjo.2021.484

Aims. The South London and Maudsley High Support Rehabilitation Team supports a cohort of 120 long-term rehabilitation patients in the densely populated London borough of Southwark.

COVID-19 has a high transmission rate and is more lethal amongst the elderly, ethnic minorities and those with comorbidities.

For these reasons, COVID-19 poses a particular challenge to our patients. Most have significant comorbidities, live communally, engage infrequently with primary care and take high-risk medications like clozapine. Many are from black and minority ethnic backgrounds.

During the Spring coronavirus wave, we found that unwell patients or their carers would contact our service for advice ahead of 111, primary care or emergency services.

In response we designed a standard operating procedure to guide our response to possible cases. This aimed to ensure our advice and management for patients drew upon the latest emerging evidence.

We audited our work and the burden of disease within our service until November 2020.

Method. At a team level, we introduced same-day remote assessments structured around a standard operating procedure incorporating the latest primary care and national guidelines.
At a trust level, treatment guidelines were amended permitting consultant discretion when deciding whether an urgent blood count was required for those unwell on clozapine, and routine blood count monitoring was extended to 3 months for eligible patients

Result. By November 2020 we had only one confirmed case of COVID-19 on our caseload. This patient required ITU and recovered. Seven patients were judged 'suspected' to have suffered COVID-19 and eight were possible cases. One supported living accommodation had a possible outbreak.

Conclusion. We are surprised to have had just one confirmed case of COVID-19, despite the vulnerability of our cohort. The attentiveness of our patients and their carers to government guidelines will have contributed to this figure. They have shown remarkable resilience.

This pandemic has prompted trust-wide changes to clozapine monitoring and perhaps a permanently less intensive monitoring regime for some patients.

That our patients contacted our team ahead of 111, primary care or emergency services may reflect the close trust they place in us to support them through difficulty. It is fitting for a service aiming to provide holistic care that our scope should have expanded in this way during the pandemic. Community rehabilitation services are well placed to act as first responders.

\section{Establishing safety huddles on a general adult acute psychiatric ward: staff's views and relation with restrictive practice}

Sidra Chaudhry* and Nicoletta Lekka

Sheffield Health and Social Care NHS Foundation Trust

${ }^{*}$ Corresponding author.

doi: 10.1192/bjo.2021.485

Aims. To establish Safety Huddles (SH) on an acute general adult psychiatric ward, exploring links to restrictive practice. Additionally, to obtain multidisciplinary staff feedback on SH's impact on their workload/wellbeing and on patient care, and to identify barriers in implementation.

Background: A SH is a multidisciplinary daily briefing focused on patients most at risk, held at a fixed time and place, lasting max 5-10 minutes. Effective $\mathrm{SH}$ involve agreed actions, are informed by multidisciplinary staff feedback of data and provide the opportunity to appreciate and celebrate success in reducing harm. SH are a valuable team building activity, promoting situational awareness and helping with prioritising daily tasks.

Method. SH were introduced on September 2020. Templates were developed to prompt staff how to facilitate. Staff were encouraged to identify key goals and reflect on issues in the last and next 24 hours. Each participant was allocated a role, e.g. record keeping or dissemination of information. In December 2020, records of incidence of restrictive practice (numbers of restraints, seclusions and rapid tranquilisations) were obtained for the periods June-August 2020 and September-November 2020. Additionally, staff feedback was obtained through a short anonymous Survey Monkey questionnaire. It explored whether SH had an effect on patient care and staff's workload/wellbeing, and possible barriers to implementation.

Result. Comparing the two 3-month periods before and after $\mathrm{SH}$ implementation, restraint episodes were reduced from 47 to 21 , seclusion episodes from 19 to 2, and rapid tranquilisation episodes from 10 to 3 . Nine staff members responded to the feedback questionnaire. All believed $\mathrm{SH}$ had a positive impact on patient care, or had the potential to do so. Staff reported SH gave them 